\title{
Influence of integrated molecular pathology test results on real-world management decisions for patients with pancreatic cysts: analysis of data from a national registry cohort
}

\author{
David Loren ${ }^{1}$, Thomas Kowalski ${ }^{1}$, Ali Siddiqui ${ }^{1}$, Sara Jackson ${ }^{2 *}$, Nicole Toney ${ }^{2}$, Nidhi Malhotra ${ }^{3}$ and Nadim Haddad ${ }^{3}$
}

\begin{abstract}
Background: Integrated molecular pathology (IMP) approaches based on DNA mutational profiling accurately determine pancreatic cyst malignancy risk in patients lacking definitive diagnoses following endoscopic ultrasound imaging with fine-needle aspiration of fluid for cytology. In such cases, IMP 'low-risk' and 'high-risk' diagnoses reliably predict benign and malignant disease, respectively, and provide improved risk stratification for malignancy than a model of the 2012 International Consensus Guideline (ICG) recommendations. Our objective was to determine if initial adjunctive IMP testing influenced future real-world pancreatic cyst management decisions for intervention or surveillance relative to ICG recommendations, and if this benefitted patient outcomes.
\end{abstract}

Methods: Analysis of data from the previously described National Pancreatic Cyst Registry. Associations between real-world decisions (intervention vs. surveillance), ICG model recommendations (surgery vs. surveillance) and IMP diagnoses (high-risk vs. low-risk) were evaluated using $2 \times 2$ tables. Kaplan Meier and hazard ratio analyses were used to assess time to malignancy. Odds ratios (OR) for surgery decision were determined using logistic regression.

Results: Of 491 patients, 206 received clinical intervention at follow-up (183 surgery, 4 chemotherapy, 19 presumed by malignant cytology). Overall, $13 \%$ (66/491) of patients had a malignant outcome and $87 \%$ (425/491) had a benign outcome at 2.9 years' follow-up. When ICG and IMP were concordant for surveillance/surgery recommendations, $83 \%$ and $88 \%$ actually underwent surveillance or surgery, respectively. However, when discordant, IMP diagnoses were predictive of real-world decisions, with $88 \%$ of patients having an intervention when ICG recommended surveillance but IMP indicated high risk, and $55 \%$ undergoing surveillance when ICG recommended surgery but IMP indicated low risk. These IMP-associated management decisions benefitted patient outcomes in these subgroups, as $57 \%$ had malignant and $99 \%$ had benign outcomes at a median 2.9 years' follow-up. IMP was also more predictive of real-world decisions than ICG by multivariate analysis: OR 11.4 (95 \% Cl 6.0 - 23.7) versus 3.7 (2.4 - 5.8), respectively.

Conclusions: DNA-based IMP diagnoses were predictive of real-world management decisions. Importantly, when ICG and IMP were discordant, IMP influence benefitted patients by increasing confidence in surveillance and surgery decisions and reducing the number of unnecessary surgeries in patients with benign disease.

Keywords: Endoscopic-ultrasound guided fine-needle aspiration, Integrated molecular pathology, Pancreatic cyst, Pancreatic neoplasm, Patient registry, Real-world management decisions, Sendai 2012 International consensus guideline

\footnotetext{
*Correspondence: sjackson@interpacedx.com

${ }^{2}$ Interpace Diagnostics Corporation, 2515 Liberty Avenue, Pittsburgh, PA

15222, USA

Full list of author information is available at the end of the article
} 


\section{Background}

Although pancreatic cystic lesions carry an increased overall risk for malignancy, the majority have a benign course, with an estimated malignant transformation rate of $0.4 \%$ per year in cysts that are non-malignant at diagnosis $[1,2]$. International and European groups have published consensus recommendations for pancreatic cyst management based on first-line test results (cytology, imaging, fluid chemistry) [3, 4], although recommendations are based mainly on specific cyst histology, which is frequently indiscernible without surgery [5]. An evidence-based approach was recently proposed by the American Gastroenterological Association, although the authors noted that the available evidence was of very low quality $[5,6]$. Cytological analysis of samples collected by endoscopic ultrasound with fineneedle aspiration (EUS-FNA) has a high true positive rate for presence of malignancy (specificity 90-100 \%) but a low true negative rate (sensitivity $<\sim 40 \%$ ), and is often indeterminate [3, 7-10]. Given the poor survival rate for pancreatic carcinoma [11], management is often cautious, with many patients undergoing surgery, although only a minority have malignant cysts on surgical pathology [12-16]. A recent estimate derived from a pooled analysis of published literature indicated an invasive adenocarcinoma frequency of $15 \%$ (95\% confidence interval [CI], 12-18\%) in patients with any type of pancreatic cyst undergoing surgery [5]. Thus, there is a need for a diagnostic approach that can reliably distinguish cysts that are malignant or likely to become malignant from those that will remain benign, in order to better ascertain which patients require surgery.

The widely-used Sendai 2012 International Consensus Guideline (ICG) recommendations [3] are based on traditional imaging, cytology and fluid chemistry criteria. Studies based on actual patient outcomes show that the ICG criteria for surgery versus surveillance have high sensitivity for malignancy but a high false positive rate; thus a substantial number of patients undergo unnecessary surgery [17-19]. Recent estimates of mortality and morbidity associated with pancreatic cyst resection based on a pooled literature analysis indicate that surgery is not without harm, even when conducted in specialized centers; the overall morbidity rate was $30 \%$ (95\% CI, 25-35\%) and the mortality rate of $2.1 \%$ (95\% CI, 1.5-2.7 \%) was noted as a likely underestimation [5].

DNA mutational analysis of cyst fluid has identified features that correlate with malignancy, although they do not reliably predict malignancy risk when assessed individually [20]. Integrated molecular pathology (IMP) interrogates a panel of mutational changes in DNA (loss of heterozygosity mutations at 10 genomic loci, oncogene point mutation, DNA quantity) in the context of traditional first-line test results (cytology, fluid chemistry, imaging) to determine risk of malignancy
$[17,21]$, and is intended to be used as an adjunct to first-line testing in patients who are indeterminate for malignancy following EUS imaging and FNA of pancreatic cyst/duct fluid for cytology. The performance of IMP in determining malignancy risk was assessed in the National Pancreatic Cyst Registry study, which reviewed follow-up medical records of patients who had an IMP test as a component of their real-world care, and showed that the IMP diagnostic categories of 'benign (BEN)' and 'statistically indolent (SI)' reliably predicted benign outcomes and 'statistically higher risk (SHR)' and 'aggressive (AGG)' reliably predicted malignant outcomes [17]. Compared with a model of the ICG criteria, IMP diagnoses increased the accuracy of surgery versus surveillance recommendations. IMP correctly recommended surveillance in $84 \%$ of patients with benign outcomes for whom surgery was recommended based on ICG criteria, and also confirmed that ICG recommendations for surveillance were appropriate in $98 \%$ of patients (benign outcomes) [17]. IMP recommended surgery in four of six patients with malignancies that were missed by ICG criteria (i.e. lacking 'worrisome' features) [17]. The use of IMP testing as an adjunct to guideline-recommended criteria may help limit false negatives and increase confidence that surveillance at longer intervals is appropriate in most patients $[17,22]$.

Because IMP is associated with a per-patient cost that is greater than that of first-line tests alone, the economic utility of IMP has been assessed in order to determine its overall cost effectiveness. Using a Markov decision model with a third-party-payer perspective to compare management strategies, which included resecting only mucinous cysts based on guideline-recommended firstline testing alone or resecting only cysts with an AGG IMP diagnosis, Das et al. found that an IMP-guided management strategy was the most cost-effective approach that provided the greatest increase in qualityadjusted life years to patients by limiting the number of surgeries on patients with benign disease course while accurately diagnosing malignant disease [23].

The objective of this analysis was to determine if the adjunctive use of DNA-based IMP testing in the initial clinical diagnosis of cystic lesions influenced real-world pancreatic cyst management decisions at follow-up relative to the initial ICG recommendations alone, and if so, whether the changes were of benefit to patients with respect to their outcome (i.e. reduction in unnecessary surgery). We analyzed data from the National Pancreatic Cyst Registry cohort to determine the association between real-world physician decision for clinical intervention (i.e. surgery or chemotherapy), or surveillance and recommendations based on initial IMP diagnoses or a model of the traditional ICG criteria. 


\section{Methods}

\section{Study design and patient population}

This was an analysis of data from the previously described National Pancreatic Cyst Registry, which included adults who underwent EUS-FNA of a pancreatic cyst and had negative, non-diagnostic, indeterminate or acellular EUS-FNA cytology results. In these patients, IMP was performed as a part of clinical testing per the prescribing physician's standard of care [17]. After initial IMP testing, all available clinical data pertaining to the pancreatic lesion were abstracted from medical records into a database in a standardized manner, without additional interpretation. Ethical approval was obtained at each site as previously described with approval for continued data analysis described here maintained by the central IRB (Quorum Review IRB) [17].

IMP diagnosis (PancraGen ${ }^{\mathrm{TM}}$ using PathFinder ${ }^{\oplus}$, Interpace Diagnostics Corporation, Pittsburgh, PA, USA) was made according to standard operating procedures at Interpace prior to inclusion of patient information into the registry, and thus without knowledge of the patient's actual clinical outcome. Molecular analysis was performed as described previously [17, 20, 21]. In brief, quantitative molecular pathology parameters included DNA quantity/quality, presence of oncogene (KRAS) point mutation and extent of clonal expansion, and presence of tumor suppressor gene loss of heterozygosity $(\mathrm{LOH})$ and extent of clonal expansion, as determined by allelic imbalance. The following chromosomal loci were examined for $\mathrm{LOH}$ (associated genes in parentheses): 1p (CMM1, L-myc), 3p (VHL, HoGG1), 5q (MCC, APC), 9p (CDKN2A), 10q (PTEN, $M X I 1), 17 \mathrm{p}$ (TP53), 17q (NME1), 18q (DCC), 21q (TFF1 and PSEN2), and 22q (NF2). The method for determining the presence and clonal expansion of oncogene point mutations and $\mathrm{LOH}$ mutations has been described previously $[20,24]$. IMP integration of first-line test results (cytology, fluid chemistry, imaging) with these molecular parameters has been described elsewhere [17].

Patient outcomes at follow-up were determined as described previously [17]. Benign outcomes included benign or low/intermediate-grade dysplasia on surgical pathology, cyst resolution on repeat imaging, or follow-up of more than 23 months without evidence of malignancy. Malignant outcomes were malignant cytology results (unknown during IMP diagnosis), high-grade dysplasia (HGD), or clinically confirmed pancreatic cancer.

\section{Definitions of clinical intervention and surveillance decisions}

Real-world decisions were defined as clinical 'intervention' if any of the following outcomes occurred within 12 months of the index EUS-FNA: surgical report and/or surgical pathology, chemotherapy, or positive cytology. If none of the above 'intervention' conditions were met, the real-world decision was determined to be 'surveillance'. For IMP, diagnoses of SHR and AGG were categorized as 'high-risk', with BEN and SI being categorized as 'low-risk'. The ICG criteria model has been described in detail previously [17]: if there were no high-risk stigmata or worrisome features then cases were classed as a 'surveillance recommendation'; all other cases were classed a 'surgery recommendation'.

\section{Statistical analysis}

Associations of IMP diagnoses (high-risk vs. low-risk) and ICG recommendations (surgery vs. surveillance) with real-world decisions (intervention vs. surveillance) and actual patient outcomes (benign vs. malignant) were evaluated using $2 \times 2$ tables. Univariate and multivariate logistic regression models were used to determine the odds ratio (OR) for surgery decision and for malignancy; the $95 \%$ CI was estimated using the profile-likelihood method. Kaplan - Meier analysis was used to determine time to malignant event, with probability of benign outcome after IMP test shown, and the hazard ratio (HR) for risk of malignancy calculated using a multivariate Cox proportional hazards model.

\section{Results and Discussion}

This cohort comprised 492 patients who had cystic lesions indeterminate for malignancy, for whom further information has been reported previously [17]. Overall, 491 patients were included in this analysis; one patient whose outcome was death due to pancreatic cancer was excluded from the analysis due to lack of clear evidence for surveillance or surgical intervention at follow-up. Overall $19 \%$ (66/491) of patients had malignant outcome and $87 \%$ (425/491) of patients had benign outcome at follow-up. Median follow-up time for patients who underwent surveillance post initial EUS-FNA $(n=285)$ was 2.9 years (range, 1.9 - 7.7 years).

In total, $42 \%(206 / 491)$ of patients received a clinical intervention less than 1 year after initial EUS-FNA (Table 1). Although we refer here to real-world 'intervention' decisions, the majority of these were surgery decisions, as 183 patients (89\%) had definitive reports of surgery in their records. Of the remaining cases, four (2\%) received chemotherapy and 19 (9\%) had presumed intervention (e.g. surgery or chemotherapy) due to frankly

Table 1 Comparison of real-world decisions with ICG recommendations and IMP diagnoses

\begin{tabular}{lll}
\hline$N=491$ & Intervention, \% & Surveillance, \% \\
\hline Real-world decision at follow-up & 42 & 58 \\
Initial ICG recommendation & 59 & 41 \\
Initial IMP recommendation based & 19 & 81 \\
on high or low risk diagnosis & & \\
\hline
\end{tabular}


malignant cytology results recorded at follow-up. Thus, up to $98 \%$ of patients in this cohort had surgery, depending on the proportion of patients with malignant cytology undergoing surgery. It seems logical to suppose that surgery would be the goal in all of these 'intervention' cases but may not proceed in patients who are unfit for or decline surgery, and thus receive chemotherapy instead.

A comparison of real-world decisions with IMP and ICG model recommendations is shown in Table 1. The real-world decisions at follow-up were approximately half-way between the initial ICG recommendations and IMP diagnoses, with IMP recommending the lowest proportion of patients for intervention.

Ideally, there should be a close correlation between cases with malignant outcomes at follow-up and cases recommended for intervention because they are initially deemed high risk by diagnostic testing. Similarly, there should be a close correlation between cases with benign outcomes and cases recommended for surveillance because they are initially deemed low risk by diagnostic testing. The actual proportions of patients in this cohort with malignant and benign outcomes were $13 \%$ (66/491) and $87 \%$ (425/491), respectively, which closely reflect those with initial IMP high-risk diagnoses who were recommended for intervention (19 \%) and those with initial low-risk IMP diagnoses who were recommended for surveillance $(81 \%)$, respectively (Table 1).

When surveillance was indicated by both ICG recommendations and IMP low-risk diagnoses, $83 \%$ of patients actually underwent surveillance, which was the appropriate decision in the large majority as $99 \%$ of this group (161/162) had benign outcomes (Table 2). Kaplan - Meier analysis confirmed high probability of benign disease at follow-up in these patients (Fig. 1; black line). When intervention was indicated by both ICG and IMP, $88 \%$ of patients actually underwent intervention within 1 year of IMP testing, with the majority of patients benefiting as $66 \%(50 / 76)$ had malignant outcomes (Table 2). Kaplan Meier analysis confirmed low probability of benign disease at follow-up in these patients (Fig. 1; red line).

However, when ICG diagnoses and IMP recommendations were discordant, real-world clinical intervention and surveillance decisions at follow-up were more reflective of initial IMP diagnoses than initial ICG criteria recommendations. Patients were more likely to undergo intervention when IMP diagnoses indicated high risk and more likely to undergo surveillance when IMP diagnoses indicated low risk. When the ICG recommendation was surveillance but IMP diagnosis indicated high risk, $88 \%$ of patients actually had an intervention within 1 year of IMP testing, suggesting that IMP influenced the decision for intervention (Table 2). This decision was of benefit to the majority of these patients, as $57 \%$ had malignant outcomes (Table 2). Kaplan - Meier analysis confirmed that IMP high-risk diagnoses reliably predicted the need for intervention even when ICG criteria recommended surveillance: conditional HR for malignancy $=29.5 ; p<0.0001$ (Fig. 1 ; green vs. black line). IMP can therefore provide evidence for intervention need in cases where ICG criteria falsely indicate that surveillance is appropriate. Furthermore, when ICG recommended surgery but IMP diagnosis indicated low risk, $55 \%$ of patients underwent surveillance in real-life, suggesting that IMP facilitated more liberal surveillance decisions, which was of benefit to the vast majority of these patients as $99 \%$ had benign outcomes at a median of $\sim 3$ years' follow-up (Table 2). ICG did not predict the need for intervention in cases where IMP indicated low risk; conditional HR for malignancy $=2.3 ; p=0.08$ (Fig. 1; blue vs. black line).

When applied to management decisions for pancreatic cysts, IMP increases the confidence in surveillance decisions where ICG criteria frequently incorrectly indicate a need for intervention. A reduction in the number of unnecessary surgeries is a key aim of improving pancreatic cyst management, given the surgical morbidity risk, and particularly the mortality risk of $\sim 2 \%$ at even the most specialized academic institutions [5]. Thus, the information provided by the adjunctive use of IMP allows physicians to make better-informed decisions that ultimately benefit the health of their patients by more accurately balancing the mortality of pancreatic cancer with the risks of surgical resection.

While it is clear that adjuvant IMP diagnoses can provide a more complete picture of risk for malignancy than guideline criteria alone, and that the test can influence

Table 2 IMP diagnoses influenced real-world intervention and surveillance decisions that were of benefit to patients (total $N=491$ )

\begin{tabular}{|c|c|c|c|c|c|c|c|}
\hline \multirow[t]{2}{*}{$\begin{array}{l}\text { Initial ICG } \\
\text { recommendation }\end{array}$} & \multirow[t]{2}{*}{$\begin{array}{l}\text { Initial IMP } \\
\text { diagnosis }\end{array}$} & \multicolumn{3}{|c|}{$\begin{array}{l}\text { Patients who had clinical intervention } \\
\text { at follow-up in reality }\end{array}$} & \multicolumn{3}{|c|}{ Patients who had surveillance at follow-up in reality ${ }^{a}$} \\
\hline & & $n$ & Surgery rate, \% & Malignant outcome rate, $\%$ & $n$ & Surveillance rate, $\%$ & Benign outcome rate, $\%$ \\
\hline \multirow[t]{2}{*}{ Surveillance } & Low risk $(n=195)$ & 33 & 17 & 3 & 162 & 83 & 99 \\
\hline & High risk $(n=8)$ & 7 & 88 & 57 & 1 & 12 & 100 \\
\hline \multirow[t]{2}{*}{ Surgery } & Low risk $(n=202)$ & 90 & 45 & 9 & 112 & 55 & 99 \\
\hline & High risk $(n=86)$ & 76 & 88 & 66 & 10 & 12 & 100 \\
\hline
\end{tabular}

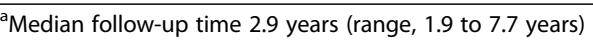




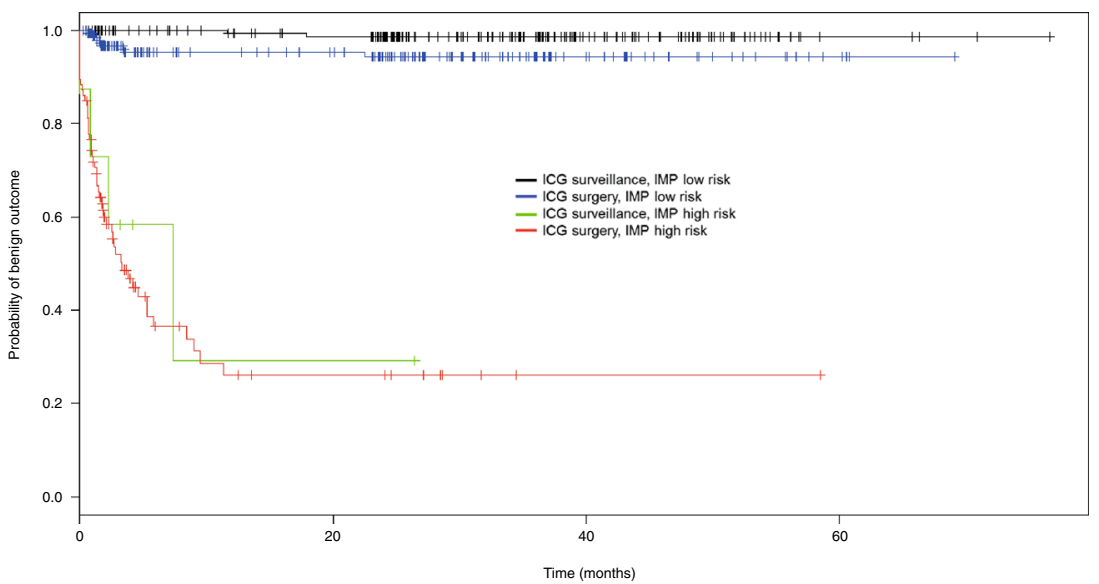

Fig. 1 Kaplan-Meier analysis of time-to-malignancy in a multivariate model as predicted by IMP and ICG. Y-axis represents probability of benign outcome in patients after initial IMP test. X-axis represents time post initial EUS-FNA that patient outcomes were determined based on review of follow-up medical records

follow-up surveillance and intervention decisions for patients, the real-world intervention rate was higher than that recommended by IMP in this cohort, although it remained lower than that recommended by ICG (Table 1). Physician clinical judgment and patient preferences play a critical role in decision making, and these factors could account for the discrepancy between the objective data derived from IMP or ICG and the decision to proceed to surgery. Additional clinical circumstances of the patient (e.g. suspicious clinical history or comorbidities, or presence of known risk factors for pancreatic cancer) are likely to influence this judgment and preference, as patients with pancreatic cysts in the real world are managed on a caseby-case basis.

Univariate analysis confirmed that initial IMP diagnoses were more predictive of actual decisions for intervention and surveillance than ICG recommendations $(\mathrm{OR}=16.8$ [95 \% CI 9.0 - 34.4] for IMP vs. 5.6 [3.7 - 8.5] for ICG), and this remained so after adjusting for ICG criteria in a multivariate analysis (Table 3 ), showing that initial IMP diagnoses were significantly predictive of real-world decisions at follow-up. IMP diagnoses were also highly predictive of actual benign and malignant patient outcomes by univariate analysis, more than traditional clinical criteria $(\mathrm{OR}=47.4$ for IMP vs. 8.5 for ICG; both $p<$ 0.0001 ), an association that remained highly significant for IMP after controlling for ICG recommendations in a multivariate analysis (adjusted $\mathrm{OR}=35.8, p<0.0001$ for IMP vs. 2.5, $p=0.066$ for ICG).

We also performed an analysis to determine if the molecular criteria that are unique to the IMP test (KRAS mutation, DNA quantity, loss of heterozygosity mutations) [17] were associated with real-world decisions for intervention and surveillance. There was a clear trend towards an association between real-world decisions for intervention in patients and high levels of each of these molecular parameters. Univariate logistic regression indicated that a combination of any 2 of these high risk molecular criteria incorporated into an IMP high-risk diagnosis was associated with real-world decisions for intervention in patients $(p=0.048)$. These findings suggest that it is this molecular component of IMP that produces the strong association between future realworld decisions and initial IMP diagnoses.

Table 3 IMP diagnoses were highly associated with real-world decisions

\begin{tabular}{|c|c|c|c|c|}
\hline \multirow[t]{2}{*}{ Association } & \multicolumn{2}{|l|}{ Univariate analysis } & \multicolumn{2}{|c|}{ Multivariate analysis } \\
\hline & OR $(95 \% \mathrm{Cl})$ & $p$ value & OR $(95 \% \mathrm{Cl})$ & $p$ value \\
\hline \multicolumn{5}{|c|}{ Real-world decision (intervention vs. surveillance) at follow-up } \\
\hline Initial ICG recommendation & $5.6(3.7-8.5)$ & $<0.0001$ & $3.7(2.4-5.8)$ & $<0.0001$ \\
\hline Initial IMP diagnosis & $16.8(9.0-34.4)$ & $<0.0001$ & $11.4(6.0-23.7)$ & $<0.0001$ \\
\hline \multicolumn{5}{|c|}{ Actual patient outcome (benign vs. malignant) at follow-up } \\
\hline Initial ICG recommendation & $8.5(3.9-22.3)$ & $<0.0001$ & $2.5(1.0-7.4)$ & 0.066 \\
\hline Initial IMP diagnosis & $47.4(23.8-102.4)$ & $<0.0001$ & $35.8(17.4-80.0)$ & $<0.0001$ \\
\hline
\end{tabular}


As discussed elsewhere, a limitation of this analysis is that the outcomes data were obtained via review of medical records from patients previously tested by IMP [17]. However, due to the benign nature of the majority of pancreatic cysts, this approach is currently the only practical method of conducting studies to assess pancreatic cyst outcomes in clinical practice. There are some minor differences between our ICG model and the published criteria, reflecting the patient cohort (those who have undergone EUS-FNA and have been prescribed IMP testing for indeterminate cystic lesions) and the study design (real-world medical record review); however, accounting for these differences were shown to have minimal impact on the performance of the ICG model in our study and no impact on the overall conclusions of the study [25].

\section{Conclusion}

In this cohort of patients with pancreatic cystic lesions indeterminate for malignancy by EUS-FNA, real-world intervention and surveillance decisions were highly associated with the initial DNA-based IMP diagnostic recommendations. Initial IMP diagnoses were more predictive of future, real-world patient management decisions and more beneficial to overall patient outcomes than solely relying on traditional EUS-FNA criteria recommended by ICG. When used as an adjuvant to traditional EUS-FNA testing, IMP increases physician confidence in the decision to proceed with surgery and reduces the number of unnecessary surgeries performed for benign disease.

\section{Abbreviations}

AGG: IMP diagnosis of 'aggressive'; BEN: IMP diagnosis of 'benign'; Cl: confidence interval; EUS-FNA: endoscopic ultrasound with fine-needle aspiration; HGD: high-grade dysplasia; HR: hazard ratio; ICG: Sendai 2012 International Consensus Guideline; IMP: integrated molecular pathology; OR: odds ratio; SHR: IMP diagnosis of 'statistically higher risk'; SI: IMP diagnosis of 'statistically indolent'.

\section{Competing interests}

This analysis was funded in part by Interpace Diagnostics Corporation. The authors received no compensation for the development of this manuscript. DL: Research grant to institution from RedPath (now Interpace) to support Registry efforts. Consultancy relationship with BSCl outside the submitted work. TK: Research grant to institution from RedPath (now Interpace) to support Registry efforts. Consultancy relationship with BSCl outside of the submitted work. AS: Research grant to institution from RedPath (now Interpace) to support Registry efforts.

SJ, NT: Employees of Interpace Diagnostics Corporation.

NM: None to disclose.

$\mathrm{NH}$ : Consultancy relationship with Boston Scientific outside of the submitted work.

\section{Authors' contributions}

TK participated in the conception and design of the Registry study; $\mathrm{DL}, \mathrm{TK}$, $\mathrm{AS}, \mathrm{NM}$, and $\mathrm{NH}$ acquired the data; DL, TK, AS, SJ, NT, NM, and NH participated in analysis and interpretation of the data; $\mathrm{DL}, \mathrm{SJ}, \mathrm{NM}$, and $\mathrm{NH}$ drafted the manuscript; all authors critically reviewed the manuscript and approved the final version. Statistical analysis was performed by an independent statistician contracted by Interpace.

\section{Acknowledgments}

Helen Varley, PhD, CMPP (Excel Scientific Solutions, Horsham, UK) provided writing assistance, which was supported by Interpace under ICMJE standards.

\section{Author details}

'Department of Medicine, Jefferson Digestive Disease Institute, Thomas Jefferson University, Philadelphia, PA, USA. ${ }^{2}$ Interpace Diagnostics Corporation, 2515 Liberty Avenue, Pittsburgh, PA 15222, USA. ${ }^{3}$ Division of Gastroenterology, MedStar Georgetown University Hospital, Washington, DC, USA.

Received: 19 October 2015 Accepted: 14 January 2016

Published online: 20 January 2016

\section{References}

1. Chernyak V, Flusberg M, Haramati LB, Rozenblit AM, Bellin E. Incidental pancreatic cystic lesions: Is there a relationship with the development of pancreatic adenocarcinoma and all-cause mortality? Radiology. 2015; 274:161-9.

2. Wu BU, Sampath K, Berberian CE, Kwok KK, Lim BS, Kao KT, et al. Prediction of malignancy in cystic neoplasms of the pancreas: a population-based cohort study. Am J Gastroenterol. 2014;109:121-9.

3. Tanaka M, Fernandez-del Castillo C, Adsay V, Chari S, Falconi M, Jang JY, et al. International consensus guidelines 2012 for the management of IPMN and MCN of the pancreas. Pancreatology. 2012;12:183-97.

4. Del Chiaro M, Verbeke C, Salvia R, Kloppel G, Werner J, McKay C, et al. European experts consensus statement on cystic tumours of the pancreas. Dig Liver Dis. 2013:45:703-11.

5. Scheiman JM, Hwang JH, Moayyedi P. American gastroenterological association technical review on the diagnosis and management of asymptomatic neoplastic pancreatic cysts. Gastroenterology. 2015;148:824-48. e22.

6. Vege SS, Ziring B, Jain R, Moayyedi P. American Gastroenterological Association Institute guideline on the diagnosis and management of asymptomatic neoplastic pancreatic cysts. Gastroenterology. 2015;148:819-22.

7. Cizginer S, Turner B, Bilge AR, Karaca C, Pitman MB, Brugge WR. Cyst fluid carcinoembryonic antigen is an accurate diagnostic marker of pancreatic mucinous cysts. Pancreas. 2011;40:1024-8.

8. Maire F, Couvelard A, Hammel P, Ponsot P, Palazzo L, Aubert A, et al. Intraductal papillary mucinous tumors of the pancreas: the preoperative value of cytologic and histopathologic diagnosis. Gastrointest Endosc. 2003;58:701-6.

9. Pitman MB, Genevay M, Yaeger K, Chebib I, Turner BG, Mino-Kenudson M, et al. High-grade atypical epithelial cells in pancreatic mucinous cysts are a more accurate predictor of malignancy than "positive" cytology. Cancer Cytopathol. 2010;118:434-40.

10. Wiersema MJ, Vilmann P, Giovannini M, Chang KJ, Wiersema LM. Endosonography-guided fine-needle aspiration biopsy: diagnostic accuracy and complication assessment. Gastroenterology. 1997;112:1087-95.

11. Siegel R, Naishadham D, Jemal A. Cancer statistics, 2013. CA Cancer J Clin. 2013;63:11-30.

12. Allen PJ, D'Angelica M, Gonen M, Jaques DP, Coit DG, Jarnagin WR, et al. A selective approach to the resection of cystic lesions of the pancreas: results from 539 consecutive patients. Ann Surg. 2006;244:572-82.

13. Grobmyer SR, Cance WG, Copeland EM, Vogel SB, Hochwald SN. Is there an indication for initial conservative management of pancreatic cystic lesions? J Surg Oncol. 2009;100:372-4.

14. Gaujoux S, Brennan MF, Gonen M, D'Angelica MI, DeMatteo R, Fong Y, et al. Cystic lesions of the pancreas: changes in the presentation and management of 1,424 patients at a single institution over a 15-year time period. J Am Coll Surg. 2011;212:590-600.

15. Huang ES, Turner BG, Fernandez-Del-Castillo C, Brugge WR, Hur C. Pancreatic cystic lesions: clinical predictors of malignancy in patients undergoing surgery. Aliment Pharmacol Ther. 2010;31:285-94.

16. Spinelli KS, Fromwiller TE, Daniel RA, Kiely JM, Nakeeb A, Komorowski RA, et al. Cystic pancreatic neoplasms: observe or operate. Ann Surg. 2004;239:651-7.

17. Al-Haddad MA, Kowalski T, Siddiqui A, Mertz HR, Mallat D, Haddad N, et al. Integrated molecular pathology accurately determines the malignant potential of pancreatic cysts. Endoscopy. 2015;47:136-46.

18. Lawson R, Savides T, Kwong W, Giap A, Hunt G, Krinsky M. Natural History of Pancreatic Cystic Lesions: Validation of the 2012 Sendai Criteria. Am J Gastroenterol. 2013;108:S91. Abstract 301.

19. Sahora K, Mino-Kenudson M, Brugge W, Thayer SP, Ferrone CR, Sahani D, et al. Branch duct intraductal papillary mucinous neoplasms: does cyst size change the tip of the scale? A critical analysis of the revised international consensus guidelines in a large single-institutional series. Ann Surg. 2013; 258:466-75. 
20. Khalid A, Zahid M, Finkelstein SD, LeBlanc JK, Kaushik N, Ahmad N, et al. Pancreatic cyst fluid DNA analysis in evaluating pancreatic cysts: a report of the PANDA study. Gastrointest Endosc. 2009;69:1095-102.

21. Shen J, Brugge WR, Dimaio CJ, Pitman MB. Molecular analysis of pancreatic cyst fluid: a comparative analysis with current practice of diagnosis. Cancer. 2009;117:217-27.

22. Kowalski T, Siddiqui A, Loren D, Mertz HR, Mallat D, Haddad N, et al. Management of patients with pancreatic cysts using integrated molecular pathology. Gastrointest Endosc. 2015;81(5):AB556-AB557.

23. Das A, Brugge W, Mishra G, Smith Jr DM, Sachdev M, Ellsworth E. Managing incidental pancreatic cystic neoplasms with integrated mutational profiling is a cost-effective strategy. Endosc Int Open. 2015;3(5):E479-E486.

24. Khalid A, McGrath KM, Zahid M, Wilson M, Brody D, Swalsky P, et al. The role of pancreatic cyst fluid molecular analysis in predicting cyst pathology. Clin Gastroenterol Hepatol. 2005:3:967-73.

25. Al-Haddad MA, Kowalski T. Reply. Gastroenterology. 2015;149:251-2.

\section{Submit your next manuscript to BioMed Central} and we will help you at every step:

- We accept pre-submission inquiries

- Our selector tool helps you to find the most relevant journal

- We provide round the clock customer support

- Convenient online submission

- Thorough peer review

- Inclusion in PubMed and all major indexing services

- Maximum visibility for your research

Submit your manuscript at www.biomedcentral.com/submit 